\title{
Oyster salinity-based habitat monitoring in Bandon Bay, Surat Thani, Thailand: A coupled eco-hydrological model approach
}

\author{
Kalanyu Sunthawanic ${ }^{\mathrm{a}}$, Kornkanok Bunwong ${ }^{\mathrm{a}, \mathrm{b}, *}$, Wichuta Sae-jie $\mathrm{e}^{\mathrm{b}, \mathrm{c}}$ \\ a Department of Mathematics, Faculty of Science, Mahidol University, Ratchathewi, Bangkok 10400 \\ Thailand \\ b Centre of Excellence in Mathematics, CHE, Bangkok 10400 Thailand \\ c Department of Applied Mathematics and Informatics, Faculty of Science and Industrial Technology, \\ Prince of Songkla University, Surat Thani Campus, Surat Thani 84000 Thailand
}

*Corresponding author, e-mail: kornkanok.bun@mahidol.ac.th

Received 16 Jul 2019

Accepted 23 Jan 2020

\begin{abstract}
Bandon Bay in Surat Thani Province, Thailand, is one of the most productive aquaculture for oysters. In order to monitor a suitable area for oyster culture related to salinity, a coupled ecological and hydrodynamical model was constructed to describe the interaction between biological and physical processes in estuarine and coastal environments. The oyster food web is modified from the classical NPZ model in which the region of attraction, the existence of equilibrium points, their asymptotic stability conditions, and the non-existence of closed orbit were analyzed. The Princeton Ocean Model with monthly mean surface wind and river discharge was employed for ocean circulation. Finally, numerical simulation has revealed the interrelation between oyster population and their salinitybased habitat, with an output that quantify the consequences for oyster density, leading to suitable oyster culture areas possibly located around the mouths of Tha Chana canal and Thatong canal.
\end{abstract}

KEYWORDS: coupled model, ecological model, hydrodynamic model, oyster, salinity

MSC2010: 00A71 34A34 34C60 65M06

\section{INTRODUCTION}

Bandon Bay is an estuary of Tapi and Phumduang rivers, located between latitude $9^{\circ} 10^{\prime}-9^{\circ} 40^{\prime} \mathrm{N}$ and longitude $99^{\circ} 20^{\prime}-99^{\circ} 60^{\prime} \mathrm{E}$, covering an estimated area of $1070 \mathrm{~km}^{2}$ in Surat Thani Province, Thailand, with the total coastline of about $80 \mathrm{~km}$ long [1]. The abundance of the estuary mainly comes from 20 subordinate rivers and channels containing nutrients and organic matters. Thai Royal Irrigation Department reported that Tapi-Phumduang river watershed is the biggest source of freshwater, releasing monthly discharge around $2 \times 10^{8} \mathrm{~m}^{3}$ in a dry season and more than $10 \times 10^{8} \mathrm{~m}^{3}$ in a wet season. These cause a huge load of phytoplankton supporting the ecosystem and aquaculture, especially the bivalve species such as oyster, cockle, and mussel. Phytoplankton is a subset of algae whose growth can be monitored via chlorophyll- $a$ level, usually varies from around $2-10 \mathrm{mg} / \mathrm{m}^{3}[2,3]$. In addition, Chumkiew et al [4] investigated the concentration of nutrients such as $\mathrm{NH}_{4}-\mathrm{N}, \mathrm{NO}_{2}, \mathrm{NO}_{3}$, and $\mathrm{PO}_{4}$ around the river mouth. The average concentration of each nutrient was approximately $0.06 \mathrm{mg} / \mathrm{l}$ in a dry season and $0.10 \mathrm{mg} / \mathrm{l}$ in a wet season. Nutrients are from agriculture fields and shrimp aquaculture flowing along the discharge. Consequently, Bandon Bay has become one of the most productive habitat of Thailand for bivalve spawning, breeding, nursery, and shelter.

Oysters are suspension-feeding bivalves important on both ecological level and economical level. They naturally inhabit in estuary and brackish water area. Suspended phytoplankton will be trapped and filtered along with the water flow by oyster's gill. C. belcheri (white scar oyster) is the main species, cultured in this bay [5]. Recently, the occurrence of heavy rain and flood crisis has caused a low salinity spread over the bay. In March 2011, there was a discharge of more than $3000 \mathrm{~m}^{3} / \mathrm{s}, 10$ times more than the average annual amount, flowed into the bay. A flood crisis may occur in November, the transition timing between the wet and the dry 
seasons, resulting in as much as 500 million baht deficit in oyster's value, as higher oyster's mortality and lower filtration rate may have arisen from lower salinity.

Oysters are in fact osmoconformer; therefore, unbalanced osmotic pressures between inside and outside body's fluid could be the cause of oyster's death. Oysters can tolerate variation in salinity but cannot expose to very low salinity or freshwater for a long period of time. Previous field research has suggested relation between oyster's mortality and salinity $[7,8]$, and Ehrich et al [6] has introduced an interrelation between oyster's filtration rate and salinity as a hyperbolic tangent function, generally displayed as an S-shape curve. Suitable salinity for oysters was recorded in the range of 15-20 psu depending on culture sites and bivalve species.

This paper is organized as follows. We first consider an ecological model of nutrient, phytoplankton, and oyster populations. Then a hydrodynamic model based on Princeton Ocean Model will be applied to the observed data to illustrate the water current and salinity variation in Bandon Bay. We finally couple the ecological model and they hydrodynamic model to monitor the effect of salinity on oyster population.

\section{ECOLOGICAL MODEL}

In this section, we will propose a system of ordinary differential equations describing the nutrientphytoplankton-oyster interactions within the homogeneous domain. For convenience, let $N(t), P(t)$, and $B(t)$ denote the nutrient concentration, the phytoplankton population, and the oyster population at time $t$, respectively.

\section{Model formulation}

The nutrient-phytoplankton-oyster interactions can be illustrated in Fig. 1. The model is based on the following assumptions. River discharge flowing into coastal region brings inputs of nutrient and phytoplankton while outflowing bay water carries out nutrients and phytoplankton [9]. Nutrients are taken up by phytoplankton while phytoplankton are grazed upon by oysters, where both of the rates are limited and can be described by the Holling type II functional response. The growths of phytoplankton and oyster depend on the presence of nutrient and phytoplankton, respectively. Moreover, the layers are thoroughly mixed at all times. Thus the dynamic of the nutrient-phytoplankton-oyster interactions can be represented by the following

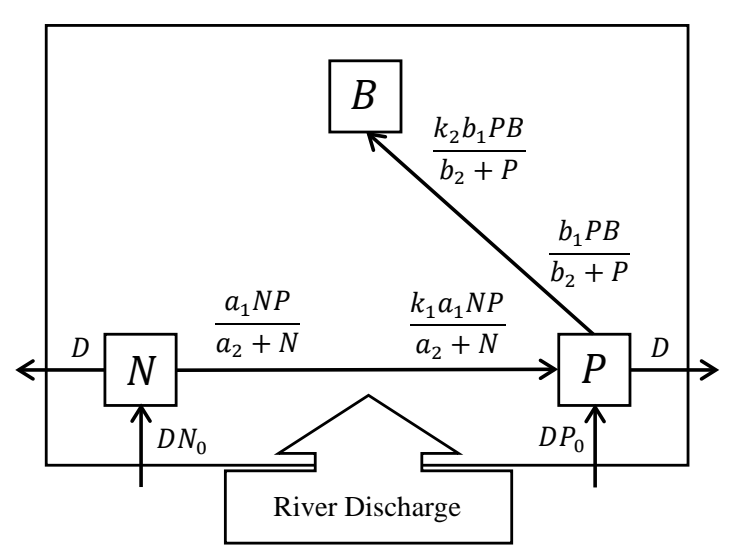

Fig. 1 A schematic diagram of oyster dynamics.

Table 1 Parameters and their ecological meanings.

\begin{tabular}{cl}
\hline Parameter & Ecological meaning \\
\hline$D$ & Water flux input and wash out rate \\
$N_{0}$ & Concentration of nutrient input \\
$P_{0}$ & Concentration of phytoplankton input \\
$a_{1}$ & Maximum specific ingestive rate of phyto- \\
& plankton \\
$a_{2}$ & Half-saturation constant of phytoplankton \\
$b_{1}$ & Maximum specific ingestive rate of oyster \\
$b_{2}$ & Half-saturation constant of oyster \\
$k_{1} \leqslant 1$ & Conversion factors from nutrient to phy- \\
& toplankton \\
$k_{2} \leqslant 1$ & Conversion factors from phytoplankton to \\
& oyster \\
\hline
\end{tabular}

All parameters are non-negative.

system of ordinary differential equations:

$$
\begin{aligned}
& \frac{\mathrm{d} N}{\mathrm{~d} t}=D\left(N_{0}-N\right)-\frac{a_{1} N P}{a_{2}+N}, \\
& \frac{\mathrm{d} P}{\mathrm{~d} t}=D\left(P_{0}-P\right)+k_{1} \frac{a_{1} N P}{a_{2}+N}-\frac{b_{1} P B}{b_{2}+P}, \\
& \frac{\mathrm{d} B}{\mathrm{~d} t}=k_{2} \frac{b_{1} P B}{b_{2}+P}-d B,
\end{aligned}
$$

where $N(0), P(0), B(0)>0$. The definitions of the parameters are summarized in Table 1 . The equations are non-dimensionalized while the meaning of parameters are preserved.

\section{Theoretical results}

Throughout this paper, $\mathbb{R}_{+}$is the set of nonnegative real number, $\mathbb{R}^{n}$ is the Euclidean $n$-space, and $\mathbb{R}_{+}^{3}$ is the nonnegative octant. The validity of system (1) is 
asserted by its uniform boundedness which will be proved in the following lemma.

Lemma 1 Suppose $\Omega:=\left\{(N, P, B) \in \mathbb{R}_{+}^{3} \mid 0 \leqslant N+P+\right.$ $B \leqslant M\}$ where $M:=D\left(N_{0}+P_{0}\right) / \eta$ and $0<\eta \leqslant$ $\min \{D, d\}$. Then all solutions of system (1) starting in $\mathbb{R}_{+}^{3}$ are uniformly bounded and $\Omega$ is the region of attraction.

Proof: Let $(N(t), P(t), B(t))$ be a solution of system (1) starting in $\mathbb{R}_{+}^{3}$ and define $W(t):=N(t)+$ $P(t)+B(t)$ and $0<\eta \leqslant \min \{D, d\}$. Therefore,

$$
\begin{aligned}
\frac{\mathrm{d} W}{\mathrm{~d} t}= & D\left(N_{0}-N\right)-\frac{a_{1} N P}{a_{2}+N}+D\left(P_{0}-P\right) \\
& +k_{1} \frac{a_{1} N P}{a_{2}+N}-\frac{b_{1} P B}{b_{2}+P}+k_{2} \frac{b_{1} P B}{b_{2}+P}-d B \\
= & D N_{0}+D P_{0}-D N-D P-d B \\
& -\left(1-k_{1}\right) \frac{a_{1} N P}{a_{2}+N}-\left(1-k_{2}\right) \frac{b_{1} P B}{b_{2}+P} \\
\leqslant & D N_{0}+D P_{0}-\eta W,
\end{aligned}
$$

where $0<\eta \leqslant \min \{D, d\}$ and $k_{1} \leqslant 1, k_{2} \leqslant 1$. Then $\frac{\mathrm{d} W}{\mathrm{~d} t}+\eta W \leqslant D N_{0}+D P_{0}$. Applying the differential inequality, we obtain

$$
0 \leqslant W(t) \leqslant D\left(N_{0}+P_{0}\right) \frac{\left(1-\mathrm{e}^{-\eta t}\right)}{\eta}+W(0) \mathrm{e}^{-\eta t} .
$$

Consequently,

$$
\limsup _{t \rightarrow \infty} W(t) \leqslant M,
$$

where $M:=D\left(N_{0}+P_{0}\right) / \eta$. It implies that all solutions of system (1) starting in $\mathbb{R}_{+}^{3}$ eventually enter the region $\Omega$.

We now discuss the existence of nonnegative equilibrium points which are the only feasible solutions for ecological models.

Lemma 2 System (1) has the following equilibrium points:

(i) The oyster-free equilibrium point $E_{1}\left(N_{1}^{*}, P_{1}^{*}, 0\right)$ always exists, where

$$
\begin{aligned}
& N_{1}^{*}=N_{0}+\frac{-\alpha_{1} N_{0}+\beta_{1}-\sqrt{\left(-\alpha_{1} N_{0}+\beta_{1}\right)^{2}+\gamma_{1}}}{2 \alpha_{1}}, \\
& P_{1}^{*}=P_{0}+k_{1}\left(N_{0}-N_{1}^{*}\right),
\end{aligned}
$$

with $\alpha_{1}=a_{1} k_{1}-D, \beta_{1}=a_{2} D+a_{1} P_{0}$, and $\gamma_{1}=$ $4 \alpha_{1} a_{1} N_{0} P_{0}$.

(ii) If the conditions $k_{2} b_{1}-d>0$ and $P_{1}^{*}>$ $d b_{2} /\left(k_{2} b_{1}-d\right)$ are satisfied, then a coexistence equilibrium point $E_{2}\left(N_{2}^{*}, P_{2}^{*}, B_{2}^{*}\right)$ exists, where

$$
\begin{aligned}
& P_{2}^{*}=\frac{d b_{2}}{k_{2} b_{1}-d}, \\
& N_{2}^{*}=N_{0}+\frac{-N_{0}-a_{2}-\frac{a_{1} P_{2}^{*}}{D}+\sqrt{\Delta}}{2}, \\
& B_{2}^{*}=\frac{k_{2} D}{d}\left[k_{1}\left(N_{0}-N_{2}^{*}\right)+\left(P_{0}-P_{2}^{*}\right)\right],
\end{aligned}
$$

with $\Delta:=\left(N_{0}+a_{2}+\frac{a_{1} P_{2}^{*}}{D}\right)^{2}-4 \frac{a_{1} N_{0} P_{2}^{*}}{D}$.

Proof: All equilibrium points $E\left(N^{*}, P^{*}, B^{*}\right)$ of system (1) could be obtained by setting (1) to zero,

$$
\begin{aligned}
D\left(N_{0}-N^{*}\right)-\frac{a_{1} N^{*} P^{*}}{a_{2}+N^{*}} & =0, \\
D\left(P_{0}-P^{*}\right)+k_{1} \frac{a_{1} N^{*} P^{*}}{a_{2}+N^{*}}-\frac{b_{1} P^{*} B^{*}}{b_{2}+P^{*}} & =0, \\
k_{2} \frac{b_{1} P^{*} B^{*}}{b_{2}+P^{*}}-d B^{*} & =0 .
\end{aligned}
$$

Solving the third equation of (2), we obtain

$$
B^{*}=0 \quad \text { or } \quad k_{2} \frac{b_{1} P^{*}}{b_{2}+P^{*}}-d=0 .
$$

For the case $B^{*}=0$, System (2) reduces to the following two dimensional nonlinear system

$$
\begin{aligned}
D\left(N_{0}-N^{*}\right)-\frac{a_{1} N^{*} P^{*}}{a_{2}+N^{*}} & =0, \\
D\left(P_{0}-P^{*}\right)+k_{1} \frac{a_{1} N^{*} P^{*}}{a_{2}+N^{*}} & =0 .
\end{aligned}
$$

Solving system (3), we obtain the oyster-free equilibrium point $E_{1}\left(N_{1}^{*}, P_{1}^{*}, 0\right)$, where

$$
\begin{aligned}
& N_{1}^{*}=N_{0}+\frac{-\alpha_{1} N_{0}+\beta_{1}-\sqrt{\left(-\alpha_{1} N_{0}+\beta_{1}\right)^{2}+\gamma_{1}}}{2 \alpha_{1}}, \\
& P_{1}^{*}=P_{0}+k_{1}\left(N_{0}-N_{1}^{*}\right),
\end{aligned}
$$

with $\alpha_{1}=a_{1} k_{1}-D, \beta_{1}=a_{2} D+a_{1} P_{0}$, and $\gamma_{1}=$ $4 \alpha_{1} a_{1} N_{0} P_{0}$.

For the case $k_{2} b_{1} P^{*} /\left(b_{2}+P^{*}\right)-d=0$, we obtain the coexistence equilibrium point $E_{2}\left(N_{2}^{*}, P_{2}^{*}, B_{2}^{*}\right)$,

$$
P_{2}^{*}=\frac{d b_{2}}{k_{2} b_{1}-d} .
$$

It is a nonnegative solution if $k_{2} b_{1}-d>0$. Consequently, system (3) reduces to the following two dimensional nonlinear system

$$
\begin{aligned}
D\left(N_{0}-N_{2}^{*}\right)-\frac{a_{1} N_{2}^{*} P_{2}^{*}}{a_{2}+N_{2}^{*}} & =0, \\
D\left(P_{0}-P_{2}^{*}\right)+k_{1} \frac{a_{1} N_{2}^{*} P_{2}^{*}}{a_{2}+N_{2}^{*}}-\frac{d B_{2}^{*}}{k_{2}} & =0 .
\end{aligned}
$$


Substituting $P_{2}^{*}$ in the first equation of (4), we obtain the nonnegative solution

$$
N_{2}^{*}=N_{0}+\frac{-N_{0}-a_{2}-\frac{a_{1} P_{2}^{*}}{D}+\sqrt{\Delta}}{2},
$$

with $\Delta:=\left(N_{0}+a_{2}+\frac{a_{1} P_{2}^{*}}{D}\right)^{2}-4 \frac{a_{1} N_{0} P_{2}^{*}}{D}$. Thus,

$$
B_{2}^{*}=\frac{k_{2} D}{d}\left[k_{1}\left(N_{0}-N_{2}^{*}\right)+\left(P_{0}-P_{2}^{*}\right)\right],
$$

which is nonnegative because $P_{2}^{*}<P_{1}^{*}$.

As a conclusion, system (1) contains two equilibrium points. To check the asymptotic stability conditions for these equilibrium points, we will determine the eigenvalues of the Jacobian matrix.

Theorem 1 The equilibrium points of system (1) have the following stability conditions:

(i) The oyster-free equilibrium point is asymptotically stable if

$$
P_{1}^{*}<\frac{d b_{2}}{k_{2} b_{1}-d} .
$$

(ii) The coexistence equilibrium point is asymptotically stable if

$$
k_{2} b_{1}-d>0 \quad \text { and } \quad P_{1}^{*}>\frac{d b_{2}}{k_{2} b_{1}-d} .
$$

Proof: After linearizing system (1) about an equilibrium point, we find that the Jacobian matrix $J$ at an equilibrium point $\left(N^{*}, P^{*}, B^{*}\right)$ can be written as

$$
J=\left[\begin{array}{ccc}
-D-\frac{a_{1} a_{2} P^{*}}{\left(a_{2}+N^{*}\right)^{2}} & -\frac{a_{1} N^{*}}{a_{2}+N^{*}} & 0 \\
\frac{k_{1} a_{1} a_{2} P^{*}}{\left(a_{2}+N^{*}\right)^{2}} & -D+\frac{\left.k_{1} a_{1}\right)^{*}}{a_{2}+N^{*}}-\frac{b_{1} b_{2} B^{*}}{\left(\frac{k_{2}}{\left.a_{2}+P^{*}\right)^{2}}\right.} & -\frac{b_{1} P^{*}}{b_{2}+p^{*}} \\
0 & \frac{k_{2} b_{1} b_{2} B^{*}}{\left(b_{2}+P^{*}\right)^{2}} & \frac{k_{2} b_{1} P^{*}}{b_{2}+P^{*}}-d
\end{array}\right] \text {. }
$$

For the equilibrium point $E_{1}\left(N_{1}^{*}, P_{1}^{*}, 0\right)$, the corresponding characteristic equation is

$$
\left(\lambda-A_{1}\right)\left(\lambda^{2}+A_{2} \lambda+A_{3}\right)=0,
$$

where

$$
\begin{aligned}
A_{1}= & \frac{k_{2} b_{1} P_{1}^{*}}{b_{2}+P_{1}^{*}}-d, \\
A_{2}= & D+\frac{a_{1} a_{2} P_{1}^{*}}{\left(a_{2}+N_{1}^{*}\right)^{2}}+D-\frac{k_{1} a_{1} N_{1}^{*}}{a_{2}+N_{1}^{*}}, \\
A_{3}= & \left(D+\frac{a_{1} a_{2} P_{1}^{*}}{\left(a_{2}+N_{1}^{*}\right)^{2}}\right)\left(D-\frac{k_{1} a_{1} N_{1}^{*}}{a_{2}+N_{1}^{*}}\right) \\
& +\left(\frac{a_{1} N_{1}^{*}}{a_{2}+N_{1}^{*}}\right)\left(\frac{k_{1} a_{1} a_{2} P_{1}^{*}}{\left(a_{2}+N_{1}^{*}\right)^{2}}\right) .
\end{aligned}
$$

Thus, the equilibrium point is locally asymptotically stable if $A_{1}<0$, i.e.,

$$
P_{1}^{*}<\frac{d b_{2}}{k_{2} b_{1}-d} .
$$

For the equilibrium point $E_{2}\left(N_{2}^{*}, P_{2}^{*}, B_{2}^{*}\right)$, the corresponding characteristic equation is

$$
\lambda^{3}+B_{1} \lambda^{2}+B_{2} \lambda+B_{3}=0,
$$

where

$$
\begin{aligned}
B_{1}= & D+\frac{a_{1} a_{2} P_{2}^{*}}{\left(a_{2}+N_{2}^{*}\right)^{2}}+D-\frac{k_{1} a_{1} N_{2}^{*}}{a_{2}+N_{2}^{*}}+\frac{b_{1} b_{2} B_{2}^{*}}{\left(b_{2}+P_{2}^{*}\right)^{2}}, \\
B_{2}= & \left(\frac{b_{1} P_{2}^{*}}{b_{2}+P_{2}^{*}}\right)\left(\frac{k_{2} b_{1} b_{2} B_{2}^{*}}{\left(b_{2}+P_{2}^{*}\right)^{2}}\right)+\left(\frac{a_{1} N_{2}^{*}}{a_{2}+N_{2}^{*}}\right)\left(\frac{k_{1} a_{1} a_{2} P_{2}^{*}}{\left(a_{2}+N_{2}^{*}\right)^{2}}\right) \\
& +\left(D+\frac{a_{1} a_{2} P_{2}^{*}}{\left(a_{2}+N_{2}^{*}\right)^{2}}\right)\left(D-\frac{k_{1} a_{1} N_{2}^{*}}{a_{2}+N_{2}^{*}}+\frac{b_{1} b_{2} B_{2}^{*}}{\left(b_{2}+P_{2}^{*}\right)^{2}}\right), \\
B_{3}= & \left(D+\frac{a_{1} a_{2} P_{2}^{*}}{\left(a_{2}+N_{2}^{*}\right)^{2}}\right)\left(\frac{b_{1} P_{2}^{*}}{b_{2}+P_{2}^{*}}\right)\left(\frac{k_{2} b_{1} b_{2} B_{2}^{*}}{\left(b_{2}+P_{2}^{*}\right)^{2}}\right) .
\end{aligned}
$$

Using Routh-Hurwitz criterion, we obtain the stability conditions $B_{1}>0, B_{3}>0$, and $B_{1} B_{2}>B_{3}$, which leads to the condition $0<P_{2}^{*}<P_{1}^{*}$.

This finding shows that if the coexistence equilibrium point exists, then it is locally asymptotically stable.

\section{HYDRODYNAMIC MODEL}

The hydrodynamic Princeton Ocean Model (POM) is a set of nonlinear primitive equations with free surface elevation [10]. It has been extensively applied in marine circulation. This model will be simulated using finite difference method with splitting time step.

\section{Model formulation}

We consider a system with orthogonal Cartesian coordinates, $x$ increasing eastwards, $y$ increasing northward, and $z$ increasing vertically upwards. The free surface is located at $z=\eta(x, y, t)$ and the bottom is at $z=-H(x, y)[11] . U, V$, and $W$ are the velocities in $x, y$, and $z$, respectively. If $\mathbf{V}=(U, V)$ is the horizontal macroscopic-global velocity vector, $\boldsymbol{\nabla}$ is the horizontal gradient operator, and $W$ is the macroscopic vertical velocity, then the continuity equation will be as follows,

$$
\boldsymbol{\nabla} \cdot \mathbf{V}+\frac{\partial W}{\partial z}=0
$$

The Reynolds momentum equations are

$$
\begin{aligned}
& \frac{\partial U}{\partial t}+\mathbf{V} \cdot \nabla U+W \frac{\partial U}{\partial z}-f V=-\frac{1}{\rho_{0}} \frac{\partial P}{\partial x}+\frac{\partial}{\partial z}\left(K_{M} \frac{\partial U}{\partial z}\right)+F_{x}, \\
& \frac{\partial V}{\partial t}+\mathbf{V} \cdot \nabla V+W \frac{\partial V}{\partial z}-f U=-\frac{1}{\rho_{0}} \frac{\partial P}{\partial y}+\frac{\partial}{\partial z}\left(K_{M} \frac{\partial V}{\partial z}\right)+F_{y},
\end{aligned}
$$


where $f$ is the Coriolis parameter, $\rho_{0}$ is the reference density, $P$ is the pressure, $K_{M}$ is the vertical eddy diffusivity of turbulent momentum mixing, and $F_{x}$ and $F_{y}$ are the horizontal diffusion terms. The conservation equation for salinity could be written as

$$
\frac{\partial S}{\partial t}+\mathbf{V} \cdot \nabla S+W \frac{\partial S}{\partial z}=\frac{\partial}{\partial z}\left(K_{H} \frac{\partial S}{\partial z}\right)+F_{s},
$$

where $S$ is the salinity, $K_{H}$ is the vertical eddy diffusivity for turbulent mixing of salt, and $F_{s}$ is the horizontal diffusion terms. Then, free surface sigma-coordinate can be introduced to the system by using the transformation equation $\sigma=(z-\eta) /(H+$ $\eta)$.

The numerical scheme of POM is divided into 2 modes: external mode and internal mode. The external mode uses a short time step to calculate 2-dimensional variables, such as vertically averaged velocities and sea elevation. While the internal mode uses a longer time step to calculate 3dimensional variables, such as velocities, temperature and salinity.

\section{Model operation}

In this work, the prediction of POM with appropriate boundary conditions is used to indicate the unknown salinity and water current at the bay. In a shallow water area, the surface wind and the river discharge play more important role than the exchanged heat and salt flux between domain and atmosphere. The numerical time steps are $6 \mathrm{~s}$ for the external mode and $120 \mathrm{~s}$ for the internal mode. The model are driven from the initial state $(t=0)$ until a quasi-steady state is achieved for 1 year. The results are illustrated by using Ocean Data View software.

\section{Data input and adjustment}

In order to simulate the hydrodynamic model, we first need to assign the domain of the simulation. The boundary conditions, such as sea elevation, wind stress and river discharge, are then applied to the model. A fine adjustment of parameters and inputs might be needed for improved results.

The computational domain covers the whole Bandon Bay, represented as a set of uniform grids as shown in Fig. 2. Lateral boundaries coincide with vertical planes. Four river discharges pass through the grids while the northern open boundary is connected to the sea. The model consists of $63 \times 37$ spatial grids of approximately $0.55^{2} \mathrm{~km}^{2}$ each with 7 layers. The bottom depth of each grid is determined from the nautical chart. The bathymetry of the

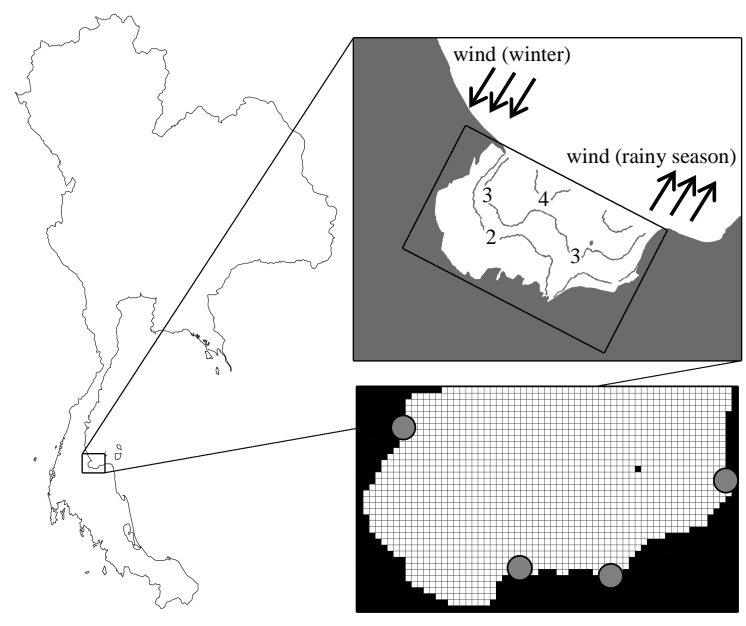

Fig. 2 Bandon Bay's bottom depths with approximate wind direction and model grid. White and black colors indicate water and land grids, respectively. Gray circles on model grid represent discharge grids at the river mouths.

domain is supposed to be $1.5 \mathrm{~m}$ depth MSL (mean sea level) along the coastal areas and up to $5 \mathrm{~m}$ depth MSL around the areas exposed to the open sea.

The boundary conditions of the model are assigned as follows. For the sea elevation boundary, there is a sea level monitoring station at Koh Prap, situated inside the bay. According to its tidal chart, we calculate the tidal constituents of the 4 major tides which are dominant in the Gulf of Thailand, namely $M_{2}, S_{2}, K_{1}$, and $O_{1}$ [12]. As a result, $A_{M_{2}}=0.21 \mathrm{~m}, P_{M_{2}}=95.5^{\circ}, A_{S_{2}}=0.13 \mathrm{~m}, P_{S_{2}}=$ $261.1^{\circ}, A_{K_{1}}=0.37 \mathrm{~m}, P_{K_{1}}=93.7^{\circ}, A_{O_{1}}=0.24 \mathrm{~m}$, and $P_{O_{1}}=347.2^{\circ}$, where $A$ and $P$ are the amplitude and the phase of each tide, respectively. Hence, to avoid a negative value of sea level during low tide, the minimum depth is set at $1.5 \mathrm{~m}$. Moreover, the boundary conditions for elevation are defined by using these 4 tides in the external mode with a radiation condition for velocity. From the cotidal charts of the Gulf of Thailand [12], we assume that the phase of each tide increases from the western boundary to the eastern boundary with a constant amplitude. For the internal time step, a radiation condition is also applied [10]. The bottom boundary conditions of sigma-coordinate are given by

$$
\frac{K_{M}}{D}\left(\frac{\partial u}{\partial \sigma}, \frac{\partial v}{\partial \sigma}\right)=\frac{\kappa^{2}}{\left[\ln \left(z_{\mathrm{bot}} / z_{0}\right)\right]^{2}} \sqrt{u^{2}+v^{2}}(u, v),
$$

where $K_{M}$ is the vertical viscosity, $D$ is thewater depth, $\kappa=0.4$ is the von Kerman constant, $z_{0}=0.01$ 
is the bottom roughness, and $z_{\mathrm{bot}}$ is half the height of the bottom layer.

We calculate the wind stress, then assign it to the surface boundary conditions. According to Thai Meteorological Department, Thailand officially enters the winter season (November-January) when the northeast monsoon occurs, and the rainy season (May-September) when the southwest monsoon occurs. The average wind speed of each month is used to calculate the surface wind stress. Due to low wind speed on the domain (3-6 m/s), we define a drag coefficient $C_{d}=0.0012$ and calculate the surface wind stress of the surface boundary from the equation

$$
\left(\tau_{x}, \tau_{y}\right)=\rho_{\text {air }} C_{d} \sqrt{u^{2}+v^{2}}(u, v),
$$

where $\rho_{\text {air }}=1.22$ is the air density, $u$ and $v$ are the wind speed in the $x$ and $y$ directions, respectively.

As for the grid boundary of the river, Tapi and Phumduang rivers are the major sources of freshwater among many rivers and channels. According to the average discharge of Tapi-Phumduang River watershed from Royal Irrigation Department, in normal situation, the lowest discharge is $150 \mathrm{~m}^{3} / \mathrm{s}$ in February, and the highest one is $600 \mathrm{~m}^{3} / \mathrm{s}$ in October. Tapi and Phumduang rivers are located on the coast, represented by the lower gray circles in Fig. 2. In addition, the left and the right gray circles represent Tha Chana canal and Thatong canal, respectively, which, based on their cross sections, presumably account for half of the amount released by Tapi river. According to Kourafalou et al [13], at the river grids, the discharges are transformed into the water elevation in the numerical process and flow to the surrounded grids at each time step. Nevertheless, an abnormal heavy rain may occasionally occur, causing a flood crisis in Surat Thani Province. To simulate such flood event, a discharge of $1200 \mathrm{~m}^{3} / \mathrm{s}$, an amount double of the highest normal value, will be assigned to Tapi and Phumduang rivers. Tha Chana canal and Thatong canal will be assigned in the same manner as Tapi and Phumduang rivers.

To initiate the salinity variation, the observed data will be interpolated. Additionally, the annual highest near-boundary salinity (27 psu) was used as the boundary conditions in each time step.

There are 7 data collection points located around the bay which represent both coastal areas and offshore areas. The salinity data was collected in July 2017, November 2017, and March 2018 for a purpose of numerical comparison. Table 2 shows
Table 2 Average salinity data ( $p s u$ ) from July 2017 to March 2018.

\begin{tabular}{lccc}
\hline & July 2017 & November 2017 & March 2018 \\
\hline Coastal Area & 17.95 & 20.00 & 26.01 \\
Offshore Area & 21.37 & 22.83 & 26.75 \\
\hline
\end{tabular}

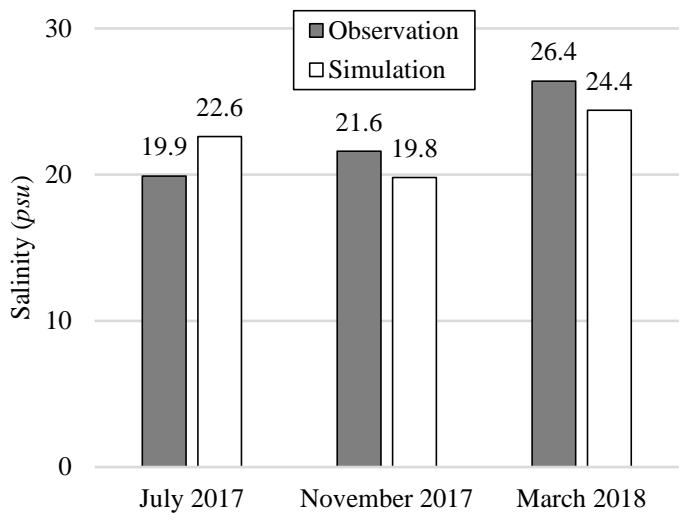

Fig. 3 The comparison of average salinity between observed data and numerical results.

the average observed salinity. Some data may be improper for comparison because of an unexpected event, for instance, a whole-week rainfall shortage in November which generally leads to higher salinity.

In fact, some coastal areas become dry grid during low tide. Hence, the depletion of freshwater and the distribution of salinity in the simulation might be lower than expectation which affect the oyster population dynamic. In a first attempt, the salinity in coastal areas is lower than that of the observed data. Therefore, we adjust the salinity boundary and river discharge using 3 criteria: small error comparing to observed data, small deviation of water current from the first attempt, and the suitable salinity range for coupled model. Thus, 25 psu boundary salinity and half amount of normal river discharge are assigned. All results afterwards are generated using these boundary values.

In normal situation, the salinities from the observed data and from the numerical results are compared in Fig. 3. The simulated salinity is 2.0 psu lower than the observed salinity in March, but is higher in July. In November, the different salinities between the simulation and observation is $1.1 \mathrm{psu}$ in the offshore areas and $3.6 \mathrm{psu}$ in the coastal areas. Lastly, the absolute error of the salinity comparison is 2.5 psu. Fig. 4 and Fig. 5 illustrate 


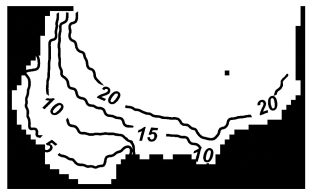

(a)
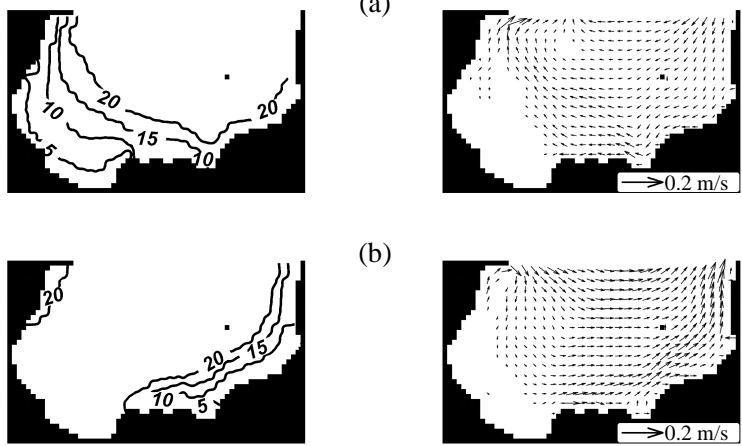

(b)

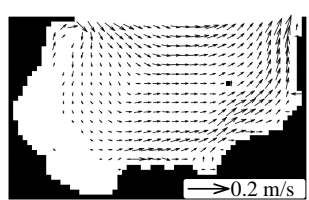

(c)
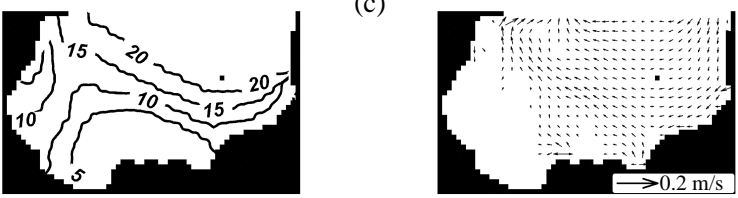

Fig. 4 Simulation of average monthly salinity (left) and water current (right) in normal situation: (a), (b), and (c) represent the results in March, July, and November, respectively.

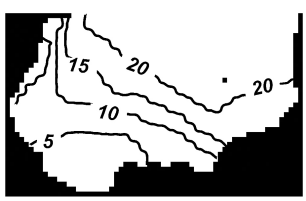

(a)
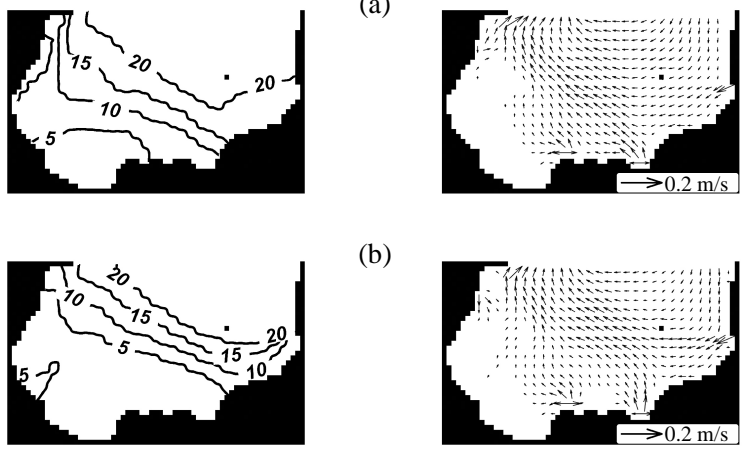

(b)

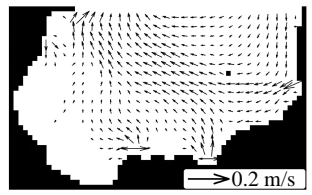

Fig. 5 Simulation of average monthly salinity (left) and water current (right) in flood situation: (a) and (b) represent the results in March and November, respectively.

the water current and salinity variation in 3 different situations: normal situation, flooding in March, and flooding in November.

\section{COUPLED MODEL}

According to Ehrich et al [6], oyster's consumption rate with salinity parameter $S(t)$ and the oyster's filtration rate $b_{1}$, can be modified as

$$
b_{1}=b_{1}(S(t))= \begin{cases}0, & S(t) \leqslant s_{1}, \\ \frac{\beta\left(S(t)-s_{1}\right)}{s_{2}-s_{1}}, & s_{1}<S(t)<s_{2}, \\ \beta, & S(t) \geqslant s_{2},\end{cases}
$$

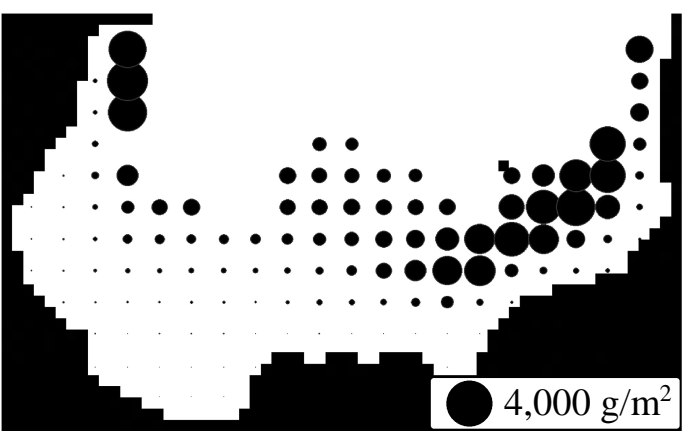

Fig. 6 Oyster's density depended on location at the end of second year.

where $s_{1}$ is the first salinity threshold of the filtration, $s_{2}$ is the second threshold, and $\beta$ is the maximum growth rate. From this equation, the oyster's growth is not affected by high salinity whereas the oyster's filtration is ceased in low salinity situation.

From La Peyre et al and Verween et al $[7,8]$, the oyster mortality rate can be described as a parabolic function of salinity

$$
d=d(S(t))=\delta_{1}+\delta_{2}\left(S(t)-s_{d}\right)^{2},
$$

when $\delta_{1}$ is the minimal death rate, $\delta_{2}$ is related to tolerant range of oyster to salinity, and $s_{d}$ is the suitable salinity for oyster's death rate. From previous observations, $s_{d}$ is seemingly greater than $s_{2}$.

In order to couple the models, we first use the same ecological model in each grid. The output of hydrodynamic model is used as the input of the ecological model without feedback from ecological part to the hydrodynamic part [14]. Fig. 6 and Fig. 7 show the numerical results of system (1) for parameters $a_{1}=3, a_{2}=200, b_{2}=10, k_{1}=0.8, k_{2}=$ $0.6, \beta=0.01, s_{1}=3, s_{2}=10, \delta_{1}=0.0001, \delta_{2}=$ $0.0001, s_{d}=18$ with $30 \mathrm{~s}$ time step, for which the coexistence equilibrium point is asymptotically stable. The boundary values of river grids is $\left(N_{0}, P_{0}\right)=$ $(0.300,0.001) \mathrm{g} / \mathrm{m}^{3}$ and the outer sea is $\left(N_{0}, P_{0}\right)=$ $(0.005,0.005) \mathrm{g} / \mathrm{m}^{3}$. The oyster cultivation grids are distributed along the grid with the depth of less than $3 \mathrm{~m}$, and initial oyster density of $1000 \mathrm{~g} / \mathrm{m}^{2}$. The period of simulation is 2 years, at which oysters have reached the market size.

\section{DISCUSSION AND CONCLUSION}

The theoretical results from the ecological model imply that oysters tend to survive in the environment with high nutrient and phytoplankton fluxes. 


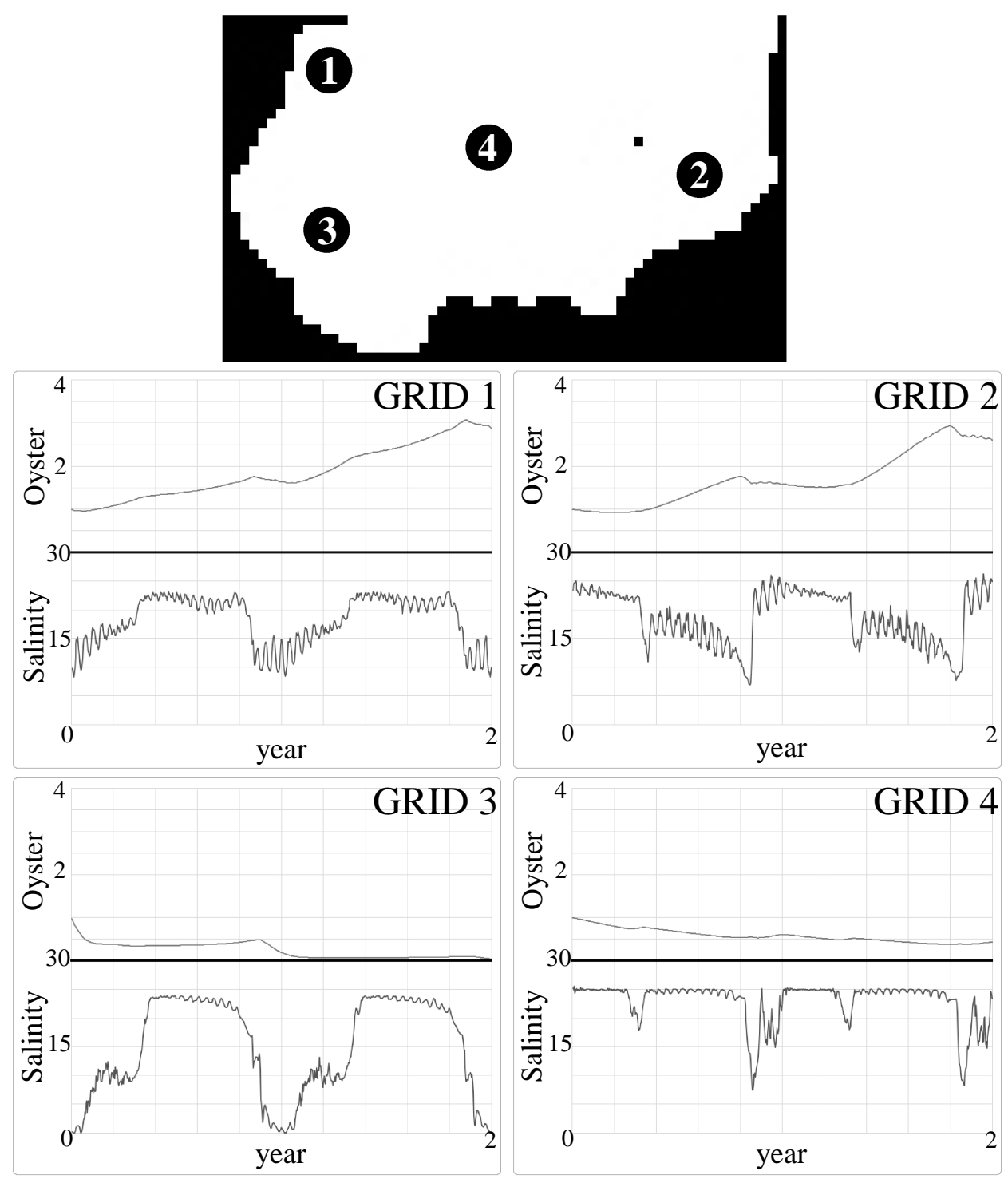

Fig. 7 The comparison of the time series of oyster density $\left(\times 10^{3} \mathrm{~g} / \mathrm{m}^{2}\right)$ and salinity $(\mathrm{psu})$ at 4 selected grids.

Moreover, an increase of oyster density in each grid may be influenced by the combination between low mortality rate and high filtration rate.

The numerical results are as follows. In normal situation, the wind play an important role on the salinity variation. The southwest wind causes a high-speed water current to the right of the bay. In July, the average water current is $0.027 \mathrm{~m} / \mathrm{s}$ in counter-clockwise direction with $21 \mathrm{psu}$ average salinity. High-speed water current causes a decrease in the freshwater residence time as well as an increase in the salinity in the bay. Even though the river discharge in July is higher than in March, the average salinity in July is higher due to the water current.

The northeast wind in winter causes an opposite direction of water current compared to the southwest wind. The average water current in November is only $0.016 \mathrm{~m} / \mathrm{s}$ with a clockwise direction inside the bay and the average salinity is $13 \mathrm{psu}$. In March, the average water current is $0.019 \mathrm{~m} / \mathrm{s}$ with $18 \mathrm{psu}$ average salinity because the river discharge is lower than that in November and wind does not totally change. In winter, freshwater tends to flow along the coastline to the left of the bay before heading out to the sea. 
Surprisingly, the average water current at the lower-left of the bay is the lowest. Moreover, the magnitude of water current in this areas is low, comparing to other areas. Hence, freshwater or sea water residence time is longer than other parts of the bay. Even though the nutrient and phytoplankton can be accumulated, but this area might not be a good place for oyster culture due to low-speed water current and long water's residence time.

At the end of the second year, the most suitable places for oyster culture are around the mouth of Tha Chana canal and Thatong canal. These places have 18-19 psu in average, equivalent to the suitable salinity for oyster's death rate. Noticeably, they are not too far from the river mouth.

In contrast, the culture sites far away from the river mouth are not suitable for oyster culture because of high salinity and lacking of phytoplankton. In another situation, the oyster culture near the river mouth may face the effect of low salinity. At the lower-left of the bay, freshwater or sea water tend to have a long residence time with low magnitude of water current, causing an extreme high or low salinity. Due to water current and freshwater usually inflow along the coastline of the bay, the salinity at the center of the bay is maintained in the highest state which matches the sea boundary salinity.

In normal situation and flood situation, the pattern of salinity variation is the same. The oyster densities in both situations are different by approximately $5 \%$. In fact, near-river mouth salinity during rainy season is $5-10 \mathrm{psu}$.

When hourly river discharge, wind inside the bay, accurate water depth, and open boundary conditions become available, the results could be improved in precision and accuracy. Interestingly, the model with wet-dry capability should be considered since oysters might expose to the air for a period of time.

Acknowledgements: This research was financially supported by (1) Department of Mathematics, Faculty of Science, Mahidol University, (2) the Centre of Excellence in Mathematics, Commission on Higher Education, and (3) Research and Researchers for Industries Scholarship, Thailand Research Fund. For the hydrodynamic model's input data, we would like to thank (1) Royal Irrigation Department, (2) Hydrographics Department, Royal Thai Navy, and (3) Thai Meteorological Department. Lastly, we would like to thank S. Sinma for sharing popular wisdom in Bandon Bay and A. Buranapratheprat for Princeton Ocean Model training.

\section{REFERENCES}

1. Wattayakorn G, Prapong P, Noichareon D (2001) Biogeochemical budgets and processes in Bandon Bay, Surat Thani, Thailand. J Sea Res 46, 133-142.

2. Kaewnern M, Yakupitiyage A (2008) Contribution of mollusc culture to control eutrophication in the coastal bay: a case study of Bandon Bay, Surat Thani, Thailand. Kasetsart Univ Fish Res Bull 32, 1-18.

3. Yoshikawa T, Tomizawa K, Okamoto Y, Watanabe K, Salaenoi J, Hayashizaki K, Kurokura H, Ishikawa S (2017) Nutrients, light and phytoplankton production in the shallow, tropical coastal waters of Bandon Bay, southern Thailand. Mar Ecol 38, ID e12475.

4. Chumkiew S, Jaroensutasinee K, Jaroensutasinee M (2015) Assessment of spatio-temoral variations in water quality of Bandon Bay, Thailand. Indian J Geo Mar Sci 44, 1000-1010.

5. Bussarawit S, Cedhagen T, Shirayama Y, Torigoe K (2010) Field Guide to the Oyster Fauna of Thailand, Kyoto Univ Press, Kyoto, Japan.

6. Ehrich MK, Harris LA (2015) A review of existing eastern oyster filtration rate models. Ecol Model 297, 201-212.

7. La Peyre MK, Eberline BS, Soniat TM, La Peyre JF (2013) Differences in extreme low salinity timing and duration differentially affect eastern oyster (Crassostrea virginica) size class growth and mortality in Breton Sound, LA. Estuar Coast Shelf Sci 135, 146-157.

8. Verween A, Vincx M, Degraer S (2007) The effect of temperature and salinity on the survival of Mytilopsis leucophaeata larvae (mollusca, bivalvia): The search for environmental limits. J Exp Mar Biol Ecol 348, 111-120.

9. De Angelis DL (1992) Dynamics of Nutrient Cycling and Food Webs, Chapman and Hall, London.

10. Mellor GL (2004) Users Guide for a Three Dimensional, Primitive Equation, Numerical Ocean Model, Princeton Univ Press, Princeton, NJ.

11. Blumberg AF, Mellor GL (1987) A description of a three-dimensional coastal ocean circulation model. In: Heaps NS (ed) Three-Dimensional Coastal Ocean Models 4, American Geophysical Union, pp 1-16.

12. Fang G, Kwok Y, Yu K, Zhu Y (1999) Numerical simulation of principal tidal constituents in the south china sea, Gulf of Tonkin and Gulf of Thailand. Cont Shelf Res 19, 845-869.

13. Kourafalou VH, Oey L, Wang JD, Lee TN (1996) The fate of river discharge on the continental shelf: 1. Modeling the river plume and the inner shelf coastal current. J Geophys Res 101, 3415-3434.

14. Ganju NK, Brush MJ, Rashleigh B, Aretxabaleta AL, del Barrio P, Grear JS, Harris LA, Lake SJ, et al (2016) Progress and challenges in coupled hydrodynamicecological estuarine modeling. Estuar Coasts 39, 311-332. 